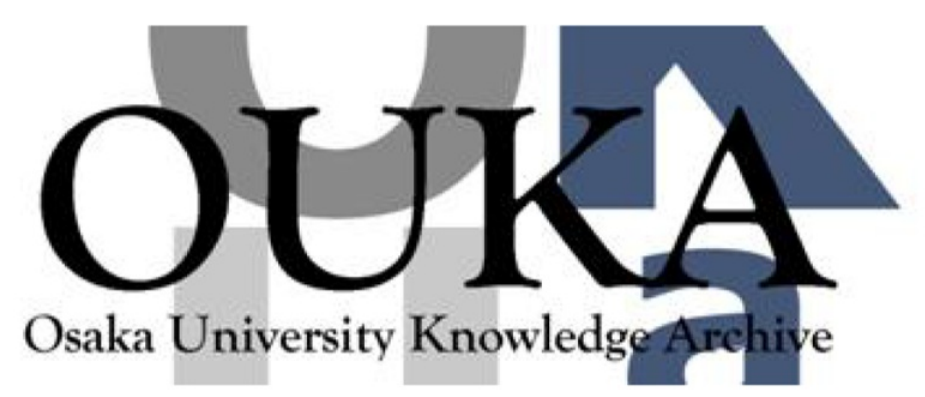

\begin{tabular}{|c|l|}
\hline Title & $\begin{array}{l}\text { Nonlinear-interferometric generation of number- } \\
\text { phase-correlated fermion states }\end{array}$ \\
\hline Author(s) & Kitagawa, Masahiro; Ueda, Masahito \\
\hline Citation & Physical Review Letters. 67(14) p. 1852-p. 1854 \\
\hline Issue Date & $1991-09-30$ \\
\hline oaire:version VoR \\
\hline URL & https://hdl.handle.net/11094/77658 \\
\hline rights & $\begin{array}{l}\text { Copyright (1991) by the American Physical } \\
\text { Society }\end{array}$ \\
\hline Note & \\
\hline
\end{tabular}

Osaka University Knowledge Archive : OUKA

https://ir. Library. osaka-u. ac. jp/

Osaka University 


\title{
Nonlinear-Interferometric Generation of Number-Phase-Correlated Fermion States
}

\author{
Masahiro Kitagawa and Masahito Ueda \\ NTT Basic Research Laboratories, Musashino, Tokyo 180, Japan \\ (Received 26 April 1991)
}

\begin{abstract}
We propose a nonlinear-interferometric scheme for generating two fermion states correlated in particle number or in wave phase. This scheme can be used for surpassing either the standard quantum limit of the number-partition noise or that of the interferometric phase sensitivity, up to a factor of the order of (total particle number $)^{1 / 3}$. A possible experimental scheme in a mesoscopic system is discussed.
\end{abstract}

PACS numbers: 42.50.Dv, 03.65.Bz, 41.80.-y

The phase sensitivity of an interferometer is improved by utilizing phase-correlated states. For optical interferometers, squeezed states are available for this purpose $[1,2]$. Yurke has pointed out that the phase sensitivity in fermion interferometers can reach $2 / N$, where $N$ is the total particle number [3]. He also gave specific states that exhibit this limit, although he did not offer any means of preparing such states. Number-correlated light beams (twin photons) have been generated using parametric fluorescence [4]. A scheme for generating numbercorrelated fermions, however, still remains to be found. In this Letter we propose a scheme for generating either number-correlated or phase-correlated fermion states. These states may be referred to as squeezed fermion states in the sense that uncertainty of a certain observable for the combined system is suppressed below the standard quantum limit.

We use a nonlinear Mach-Zehnder interferometer as shown in Fig. 1, where two quasimonochromatic fermion fields

$$
\begin{aligned}
& A(z, t)=\frac{1}{\sqrt{L}} \sum_{k} a_{k} e^{i\left(k z-\omega_{k} t\right)}, \\
& B(z, t)=\frac{1}{\sqrt{L}} \sum_{k} b_{k} e^{i\left(k z-\omega_{k} t\right)}
\end{aligned}
$$

are propagating and interacting through the interferometer. Fermion creation and annihilation operators $a_{k}^{\dagger}$ and $a_{k}$ for system $A$ and $b_{k}^{\dagger}$ and $b_{k}$ for system $B$ obey the

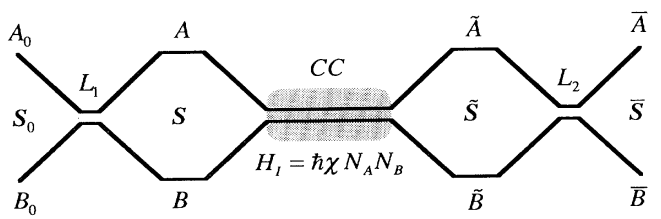

FIG. 1. A schematic illustration of the nonlinear MachZehnder interferometer with a Coulomb coupler as a correlated fermion generator. The solid lines denote paths for fermions. $\mathrm{L}_{\mathrm{l}}$ is an input linear coupler which splits an input fermion field $A_{0}$ into $A$ and $B$. CC is a Coulomb coupler which establishes a quantum-mechanical correlation between $A$ and $B . \quad \mathrm{L}_{2}$ is an output linear coupler which combines $\tilde{A}$ and $\tilde{B}$ to make suitably correlated outputs $\bar{A}$ and $\bar{B}$. anticommutation relations: $\left\{a_{k}^{\dagger}, a_{k^{\prime}}\right\}=\delta_{k k^{\prime}}, \quad\left\{a_{k}, a_{k^{\prime}}\right\}=0$, $\left\{b_{k}^{\dagger}, b_{k^{\prime}}\right\}=\delta_{k k^{\prime}},\left\{b_{k}, b_{k^{\prime}}\right\}=0,\left\{a_{k}^{\dagger}, b_{k^{\prime}}\right\}=0$, and $\left\{a_{k}, b_{k^{\prime}}\right\}=0$. $L$ denotes the quantization length. Various accents in Fig. 1 distinguish the fields and the associated operators at different points. Fermions are fed only to an input port $A_{0} . \mathrm{L}_{1}$ is an input linear coupler which divides an input fermion field $A_{0}$ into $A$ and $B$. CC is a nonlinear coupler which establishes a quantum-mechanical correlation between $A$ and $B$. The output fields of this coupler, $\tilde{A}$ and $\tilde{B}$, are combined at another linear coupler $\mathrm{L}_{2}$, yielding suitably correlated outputs $\bar{A}$ and $\bar{B}$.

The two interacting fermion systems can be expressed in terms of spin operators $S=\left(S_{x}, S_{y}, S_{z}\right)$,

$$
S_{+} \equiv \sum_{k} a_{k}^{\dagger} b_{k}, \quad S_{z} \equiv \frac{1}{2}\left(N_{A}-N_{B}\right),
$$

where $S_{ \pm}=S_{x} \pm i S_{y^{\prime}}, N_{A}=\sum_{k} a_{k}^{\dagger} a_{k}$, and $N_{B}=\sum_{k} b_{k}^{\dagger} b_{k}$. If $A_{0}$ has $N_{0}$ fermions and $B_{0}$ is in the vacuum state, the combined system $\mathbf{S}_{0}$ is in the simultaneous eigenstate of $\mathbf{S}^{2}$ and $S_{z}$

$$
\mathbf{S}^{2}|S, S\rangle=S(S+1)|S, S\rangle, \quad S_{z}|S, S\rangle=S|S, S\rangle,
$$

where $S=N_{0} / 2$ [3].

Linear couplers or beam splitters are described by the interaction Hamiltonian

$$
H_{I}=\hbar g\left(e^{-i \phi} \sum_{k} a_{k}^{\dagger} b_{k}+e^{i \phi} \sum_{k} b_{k}^{\dagger} a_{k}\right)=2 \hbar g \mathbf{n} \cdot \mathbf{S},
$$

where $g>0$ and $\mathbf{n} \equiv(\cos \phi, \sin \phi, 0)$. This interaction couples two fermion systems as

$$
\left(\begin{array}{l}
a_{k}^{\prime} \\
b_{k}^{\prime}
\end{array}\right)=\left(\begin{array}{cc}
\cos (\Omega / 2) & -i e^{-i \phi} \sin (\Omega / 2) \\
-i e^{i \phi} \sin (\Omega / 2) & \cos (\Omega / 2)
\end{array}\right)\left(\begin{array}{l}
a_{k} \\
b_{k}
\end{array}\right),
$$

where $\Omega=2 g \tau$ with $\tau$ being the interaction time. In spin notation, it corresponds to a rotation of the spin through the angle $\Omega$ about the axis $n$ lying in the $x-y$ plane,

$$
\mathbf{S}^{\prime}=\exp (i \mathbf{\Omega} \cdot \mathbf{S}) \mathbf{S} \exp (-i \mathbf{\Omega} \mathbf{n} \cdot \mathbf{S}) \text {. }
$$

Let $L_{1}$ be a $50 \%$ coupler with $\Omega=\pi / 2$ and $\mathbf{n}=\mathbf{y}$ be a unit vector pointing in the $+y$ direction. It rotates the spin about the $y$ axis through $\pi / 2$, giving an eigenstate of $S_{x}$ described by the coherent spin state $|\theta, \phi\rangle=|\pi / 2,0\rangle$ [5]. Since $\left\langle S_{z}\right\rangle=0, A$ and $B$ contain the same average 

$+N_{B}$ is a constant of motion $\left(=N_{0}\right)$. This is a conventional equal partition of a fermion source by a linear coupler. For later reference, let us find correlations in particle number and in wave phase between these two fermion systems. The correlation in particle number is measured by the standard deviation of the number difference,

$$
\delta N \equiv\left\langle\left[\Delta\left(N_{A}-N_{B}\right)\right]^{2}\right\rangle^{1 / 2}=2\left\langle\Delta S_{z}{ }^{2}\right\rangle^{1 / 2}=N_{0}^{1 / 2},
$$

while the correlation in wave phase is measured by the standard deviation of the phase difference,

$$
\delta \phi \equiv\left\langle\left[\Delta\left(\phi_{A}-\phi_{B}\right)\right]^{2}\right\rangle^{1 / 2} \approx \frac{\left\langle\Delta S_{y}{ }^{2}\right\rangle^{1 / 2}}{\left|\left\langle S_{x}\right\rangle\right|}=\frac{1}{N_{0}^{1 / 2}} .
$$

The minimum detectable phase difference of a phasesensing interferometer is limited by $\delta \phi$, which is $N_{0}^{-1 / 2}$ for a conventional interferometer using a linear coupler as an input partition device. Equations (5) and (6) are referred to as the standard quantum limit of the numberpartition noise and that of the interferometric phase sensitivity. Note that they satisfy the minimum uncertainty relationship

$$
\delta N \delta \phi=1 .
$$

We now proceed to the heart of the nonlinear interferometer, a nonlinear coupler $\mathrm{CC}$ that is described by the interaction Hamiltonian [6]

$$
H_{I}=\hbar \chi N_{A} N_{B}=\hbar \chi\left(N^{2} / 4-S_{z}^{2}\right) \text {. }
$$

This can be realized through a Coulomb interaction between charged particles such as electrons. We will refer to the device expressed by Eq. (8) as a Coulomb coupler. The unitary transformations generated by the interaction are given by

$$
\tilde{a}_{k}=a_{k} \exp \left(-i \mu N_{B}\right), \quad \tilde{b}_{k}=b_{k} \exp \left(-i \mu N_{A}\right),
$$

where $\mu=\chi \tau$. The field $A$ undergoes a phase shift proportional to the particle number of $B$ and vice versa. This is referred to as the mutual phase modulation of fermions. At this stage, the quantum-mechanical correlations are established between the two systems. Remarkably, the number-phase correlations prepared at this stage can be used for the quantum nondemolition measurement of fermion numbers; this possibility, in our opinion, merits further theoretical study. They are not yet operational, however, for our present purpose. To see how they can be converted into desirable correlations, we again use the spin representation for two fermion systems. number of fermions $\left\langle N_{A}\right\rangle=\left\langle N_{B}\right\rangle=N_{0} / 2$, and $N=N_{A}$

The quadratic term $S_{z}^{2}$ in Eq. (8) twists the noise distribution of a coherent spin state around the $z$ axis, and thus generates a squeezed spin state [7]. The unitary transformation by the Coulomb coupler CC is given by

$$
\tilde{\mathbf{S}}=\exp \left(-i \mu S_{z}^{2}\right) \mathbf{S} \exp \left(i \mu S_{z}^{2}\right) \text {. }
$$

The magnitude of squeezing is characterized by parameters [8]

$$
\alpha=\frac{1}{2} N_{0} \mu, \quad \beta=\frac{1}{2} N_{0} \mu^{2} .
$$

The uncertainty in a certain spin component in the $y-z$ plane is now reduced with increasing $\alpha$ but it is neither in $S_{z}$, which denotes the difference in number, nor in $S_{y}$, which denotes the difference in phase [7].

To obtain suitably correlated fermions, the spin must be rotated about the $x$ axis. This is accomplished at the output linear coupler $\mathrm{L}_{2}$ by choosing $\mathbf{n}=\mathbf{x}$ and $\boldsymbol{\Omega}$ $=\Omega_{N, P}=\frac{1}{2}( \pm \pi / 2-\arctan \alpha)(+$ for $N,-$ for $P)$ which corresponds to the beam-splitter reflectivity

$$
R_{N, P}=\frac{1}{2}\left\{1-\left[\frac{1}{2}\left(1 \pm \frac{\alpha}{\left(\alpha^{2}+1\right)^{1 / 2}}\right)\right]^{1 / 2}\right\} .
$$

If we choose $\Omega_{N},\left\langle\Delta \bar{S}_{z}{ }^{2}\right\rangle$ is reduced and numbercorrelated fermion states are generated:

$$
\begin{aligned}
& \delta N \approx N_{0}^{-1 / 2} \mu^{-1}\left(1+N_{0}^{4} \mu^{6} / 4\right)^{1 / 2} \geq\left(\frac{4}{3} N_{0}\right)^{1 / 6}, \\
& \delta \phi \approx N_{0}^{1 / 2} \mu,
\end{aligned}
$$

where $\alpha \gg 1$ and $\beta \ll 1$. If we choose $\Omega_{P},\left\langle\Delta \bar{S}_{y}{ }^{2}\right\rangle$ is reduced and phase-correlated fermion states are generated:

$$
\begin{aligned}
& \delta \phi \approx N_{0}^{-3 / 2} \mu^{-1}\left(1+N_{0}^{4} \mu^{6} / 4\right)^{1 / 2} \geq\left(\frac{4}{3}\right)^{1 / 6} N_{0}^{-5 / 6} \\
& \delta N \approx N_{0}^{3 / 2} \mu .
\end{aligned}
$$

The term $N_{0}^{4} \mu^{6}$ in Eqs. (10) and (11) arises from the swirliness of a one-axis squeezed spin state; it imposes the limit [7] on the enhancement of the correlations. The maximum enhancement of a factor $\left(\frac{3}{4}\right)^{1 / 6} N_{0}^{1 / 3}$ is achieved at $\mu=6^{1 / 6} N_{0}^{-2 / 3}$ where the last equalities in Eqs. (10) and (11) hold. It is also noted that the minimum uncertainty relationship in Eq. (7) is almost satisfied for the correlated outputs.

Let us discuss the validity of the interaction Hamiltonian (8). Consider two quasimonochromatic charged fermion fields $A(z, t)$ and $B\left(z^{\prime}, t\right)$ along one-dimensional paths. They can be either in vacuum or confined in waveguides. If two paths are parallel, then the Coulomb interaction energy between the two fields can be written as

$$
H_{C}=\int_{-L / 2}^{L / 2} d z \int_{-L / 2}^{L / 2} d z^{\prime} \frac{e^{2} A^{\dagger}(z, t) A(z, t) B^{\dagger}\left(z^{\prime}, t\right) B\left(z^{\prime}, t\right)}{4 \pi \epsilon\left[\left(z \mp z^{\prime}\right)^{2}+d^{2}\right]^{1 / 2}},
$$

where $d$ denotes the distance between the paths, $\epsilon$ the dielectric constant, and $e$ the electron charge. The interaction length is chosen to be identical with the quantization length $L$ to correlate the entire fields. The $\mp$ signs in the denomi- 
nator correspond to copropagating and counterpropagating schemes, where the coordinates $z$ and $z^{\prime}$ are taken in the opposite directions for the latter case. $H_{C}$ consists of $H_{I}$ and the residual terms. $H_{I}$ is dominant for $d>L$; the coefficient $\chi$ approaches $e^{2} / 4 \pi \epsilon d$ as $d / L$ increases. The coefficient itself can be enhanced by decreasing $d$, while $H_{I}$ becomes less dominant as $d / L$ decreases. The counterpropagating scheme is useful to suppress the residual terms.

In a mesoscopic electron interferometer fabricated with solid waveguides, the Coulomb coupling can be enhanced by placing the two electron waveguides very close to each other, while avoiding linear coupling by separating them with an oxide insulator. This will be a promising structure for correlated fermion generators and sub-quantumlimit phase-sensing interferometers.

Although we have mainly discussed charged fermions, the principle is also applicable to other quanta or particles if the nonlinear interaction in the form of Eq. (8) is valid. The Coulomb interaction can be used for charged particles such as ions. For neutral particles such as neutrons or atoms, such nonlinear interaction is yet to be found. For photons, the interaction is known as the optical Kerr effect [6].

We have shown that a nonlinear Mach-Zehnder interferometer with a Coulomb coupler acts as a numbercorrelated or phase-correlated fermion generator. The number-correlated fermion generator can suppress the partition noise in an ultrasmall electric current and may therefore find important applications in single-electron electronics. It is possible to surpass the standard quantum limit in phase sensitivity up to a factor of $\left(\frac{3}{4}\right)^{1 / 6} N_{0}^{1 / 3}$ if we replace the input linear coupler of a conventional Mach-Zehnder interferometer with the phase-correlated fermion generator presented here. Although the enhancement factor does not reach the limit $\left(N_{0} / 4\right)^{1 / 2}$ exemplified by Yurke [3], further improvement can be expected if we use a more sophisticated nonlinear interaction such as two-axis twisting [9].

[1] C. M. Caves, Phys. Rev. D 23, 1693 (1981).

[2] For recent special issues on squeezed light, see J. Opt. Soc. Am B 4, 1450 (1987), edited by H. J. Kimble and D. F. Walls; R. Loudon and P. L. Knight, J. Mod. Opt. 34 (1987).

[3] B. Yurke, Phys. Rev. Lett. 56, 1515 (1986); Physica (Amsterdam) 151B, 286 (1988); B. Yurke, S. L. McCall, and J. R. Klauder, Phys. Rev. A 33, 4033 (1986).

[4] For a recent review, see Z. Y. Ou and L. Mandel, Quantum Opt. 2, 71 (1990).

[5] J. M. Radcliffe, J. Phys. A 4, 313 (1971); F. T. Arecchi, E. Courtens, R. Gilmore, and H. Thomas, Phys. Rev. A 6, 2211 (1972); G. J. Glauber and F. Haake, Phys. Rev. A 13, 357 (1976).

[6] N. Imoto, H. A. Haus, and Y. Yamamoto, Phys. Rev. A 34, 4025 (1986); M. Kitagawa, N. Imoto, and Y. Yamamoto, Phys. Rev. A 35, 5270 (1987).

[7] M. Kitagawa and M. Ueda (to be published).

[8] $\mu$ in this Letter is different from $\mu$ in Ref. [7] by a factor of -2 . Here, $\alpha$ and $\beta$ are defined so as to absorb the factor 2 and they have the same magnitudes as those in Ref. [7].

[9] M. Kitagawa and M. Ueda (to be published). 